\section{UNIVERSIDAD Y DEFENSA: LA EXPERIENCIA DEL INSTITUTO UNIVERSITARIO GENERAL GUTIÉRREZ MELLADO}

\author{
Isidro Sepúlveda \\ Director del Instituto Universitario \\ Gutiérrez Mellado (IUGM-UNED)
}

\section{UNIVERSITY AND DEFENCE: THE EXPERIENCE OF THE UNIVERSITY INSTITUTE GENERAL GUTIÉRREZ MELLADO}

\begin{abstract}
The backbone of the activity carried out by the University Institute "General Gutierrez Mellado", belonging to UNED, are both the research and the teaching of the main matters related to peace, to security and to defense. This Institute has two main institutional sponsors such as the Universidad Nacional de Educación a Distancia (National University of Off-Site Education) and the Defense Ministry. Both intended when founding this Institute, to contribute to the knowledge of Global Security as an instrument of peaceful coexistence of citizens and the spreading of the culture of the defense. The Institute is devoted to postgraduate courses (Doctorate or PhD, Master, Expert and University Specialist), the research on matters related to international security and the spreading of knowledge through scientist reunions, publications and further analysis in the website.
\end{abstract}

KEY WORDS: Higher Education; postgraduate courses; security and defence.

El mundo universitario y la comunidad militar han tenido desde siglos pasados una relación extraordinariamente fluida. En la España del siglo XVIII las distintas ramas de las ciencias encontraban sus elementos más señeros en las filas militares; desde la astronomía a la botánica, desde la ingeniería a las matemáticas, la transferencia de conocimiento entre los dos ámbitos - prioritariamente, del militar al civil- se realizó con suma facilidad y en dimensión creciente. Como en el resto de los países europeos, estos cauces de retroalimentación se fueron cortando a lo largo del siglo XIX como consecuencia de la profesionalización de la investigación universitaria, el desarrollo del modelo

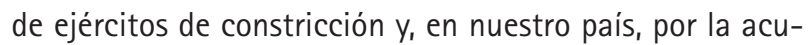
mulación de conflictos internos, el limitado desarrollo del Estado liberal y -sobre todo a partir de la Restauración canovista- el enroque endogámico de ambos colectivos. Las décadas centrales del siglo XX fueron sin duda el punto más bajo de transferencia creativa, lo que debió ser superado una vez alcanzada la normalización democrática.
RESUMEN: El Instituto Universitario General Gutiérrez Mellado, perteneciente a la UNED, tiene como eje vertebrador de su actividad la investigación y la docencia sobre los grandes temas de la paz, la seguridad y la defensa. Los dos patronos institucionales son la Universidad Nacional de Educación a Distancia y el Ministerio de Defensa, que con su fundación pretendian contribuir al conocimiento de la seguridad global como un instrumento para la pacífica convivencia entre los ciudadanos y la difusión de la cultura de la defensa. El Instituto está dedicado a los estudios de postgrado (programa de doctorado, Máster, cursos de experto y especialista universitario), la investigación sobre los amplios campos de la seguridad internacional y la difusión del conocimiento, a través de reuniones científicas, publicaciones y análisis en la página web.

PALABRAS CLAVE: Educación superior; postgrado; seguridad y defensa.

Los cambios trascendentales del final de la guerra fría y la emergencia de nuevas amenazas a la seguridad internacional han multiplicado la demanda de comunicación y colaboración. Las paulatinas reformas y la confluencia de cambios normativos en sendos ámbitos permitirán que la nueva Ley de Carrera Militar constituya el punto álgido de simbiosis entre Universidad y Fuerzas Armadas. Sin perder ninguna de las comunidades sus señas distintivas, sin abdicar de metodologías formativas decantadas por siglos de experiencia, ambos colectivos han superado rancios y esteriotipados prejuicios y desde hace años, sin esperar a la confirmación legislativa, se encuentran colaborando estrechamente, desarrollando proyectos conjuntos y alcanzando objetivos muy significativos.

En las páginas de este trabajo se presenta la actividad de un órgano surgido como fruto de esta colaboración, con entidad universitaria aunque conformado por igual por militares y civiles: el Instituto Universitario General 
Gutiérrez Mellado. El Instituto tiene como eje vertebrador de su actividad la investigación y la actividad docente sobre los grandes temas de paz, seguridad y defensa. Nació, por iniciativa del Ministerio de Defensa, precisamente para satisfacer la falta de atención sobre estas cuestiones en el ámbito universitario español, hasta entonces prácticamente ausente de una referencia que sólo podía encontrarse en los estudios de centros militares. La Universidad Nacional de Educación a Distancia acogió con entusiasmo la idea como medio para contribuir al cambio de esta situación, propiciando una manera de entender la nueva seguridad global como un instrumento para la pacífica convivencia entre los ciudadanos. El desarrollo del nuevo centro contó con la metodología pedagógica propia de la UNED, un sistema de trabajo innovador y atento a la incorporación de avances tecnológicos que potencian las posibilidades docentes en las circunstancias más variadas. Universidad y Ministerio, los dos patronos institucionales, han permanecido fieles a la idea de origen y han constituido de manera permanente el mayor apoyo y el principal impulso para las actividades del Instituto, diseñadas siempre bajo las directrices de su Patronato y con el alto respaldo del Patronato de Honor presidido por S.M. el Rey.

Difusión docente del conocimiento, análisis e investigación de los procesos abiertos y socialización de la cultura de defensa fundamentan la actividad del Instituto. El elenco de prestigiosos profesores que desarrollan su labor en el Programa de Doctorado y los cursos modulares que componen el Máster Universitario es uno de sus principales activos; este claustro constituye el nervio central de la actividad docente de calidad, que se imparte en un amplio y coherente conjunto de campos: desde la seguridad internacional a la resolución pacifica de conflictos, desde los servicios de inteligencia a la cooperación internacional. La respuesta a esta oferta docente es significativa y año tras año permanece en alza el número de alumnos que buscan en el Instituto un nivel de excelencia en los estudios de seguridad y defensa; su confianza constituye el principal estímulo en la mejora formal y de contenidos.

La proyección académica fundamental de un instituto universitario se ve reforzada por su capacidad para generar investigación de alto nivel. En los amplios campos de trabajo sobre la paz, la seguridad y la defensa, el Instituto desarrolla su investigación en tres niveles: proyectos de investigación, acuerdos de investigación y la elaboración de los trabajos académicos conducentes a la obtención del Diploma de Estudios Avanzados y el definitivo título de doctor. Los resultados de estas investigaciones, además de las conclusiones alcanzadas en los cursos y seminarios celebrados, conforman la mayor parte de la actividad editorial del Instituto.

Con ser todo ello lo más importante, el Instituto no completaría su obligación sin atender a su labor de difusor de cultura de defensa. La apertura de las actividades a una sociedad dinámica, que demanda información rigurosa y análisis profundos, exige la organización de cursos y seminarios, ciclos y encuentros, de los que el lector tiene debida cuenta en las páginas de las Memorias anuales; entre los eventos académicos destacan por su dimensión las convocatorias anuales del Congreso de Historia de la Defensa y de la Semana Iberoamericana de Seguridad y Defensa.

Dada la amplitud de estas actuaciones y las dimensiones mesurables de este trabajo, resulta más significativo realizar un estudio de caso, centraldo el análisis en el modelo docente empleado por el del Instituto Universitario General Gutiérrez Mellado (IUGM en la apócope terminología internauta), con el propósito de compartir su experiencia con otros centros de investigación y docencia. El Instituto fue creado con el propósito de implementar los recursos de la educación a distancia en el campo de las enseñanzas de posgrado en el ámbito de la Paz, la Seguridad y la Defensa. La experiencia acumulada en este campo permite exponer conclusiones y diseñar un nuevo escenario operativo, aún más innovador y ambicioso. Las posibilidades de modularidad del modelo docente y la capacidad de flexibilidad en la conformación de programas se ofrecen como una alternativa muy atractiva en el desarrollo de curricula académicos sobre estas disciplinas, participados por dos o más centros universitarios españoles, europeos y de América Latina.

\section{El Instituto Universitario General Gutiérrez Mellado}

El Instituto Universitario General Gutiérrez Mellado de Estudios sobre la Paz, la Seguridad y la Defensa fue creado por el Consejo de Ministros (Real Decreto 1643/1997), en la Universidad Nacional de Educación a Distancia, el 24 de octubre de 1997. Es el primer -y, por ahora, único- Ins- 
tituto Universitario de estas características en España y similar a instituciones ya existentes en otros países, como el Centre for Defence Studies (King's Collage, Universidad de Londres), o el Interuniversitary Seminar on Armed Forces and Society (Loyola University, Chicago), en los que se realizan trabajos de investigación sobre temas específicos relacionados con la seguridad y la defensa, además de desarrollar actividades docentes y cursos de doctorado.

La investigación y la docencia de los temas relacionados con la paz, la seguridad y la defensa ofrecen el atractivo de permitir la práctica de la interdisciplinariedad y el empleo de múltiples recursos metodológicos tanto en la investigación y la enseñanza en el ámbito de las Ciencias Sociales, como en el campo de la investigación científica y técnica de los medios utilizados al servicio de la defensa y la seguridad.

En su ámbito de estudio, el Instituto pretende cumplir una función de puente y promoción de los tres vectores de proyección internacional de España: la Unión Europea, la comunidad latinoamericana y los países del Magreb. Esta triple proyección supone una contribución importante en el ámbito cultural a los objetivos de constitución y potenciación de una España comunitaria que, sin menoscabo de su espíritu europeísta y sin olvidar las tradicionales relaciones con los países del norte de África, profundice en sus permanentes lazos de fraternidad con América Latina.

En relación con las necesidades sociales y científicas, se presta una especial atención a las necesidades de la estructura universitaria a las que el Instituto pretende responder. En primer lugar, las materias que responden a lo que genéricamente se denomina estudios sobre la paz, la seguridad y la defensa, se caracterizan por una interdisciplinariedad considerable. Las cuestiones que engloban tal denominación integran unas veintiséis áreas de conocimiento distintas, de entre las existentes en la clasificación que realiza la actual legislación universitaria; esta amplitud englobaría, por orden alfabético, desde la Antropología hasta la Sociología. La coordinación de la pluridisciplinariedad de los investigadores sobre la paz, la seguridad y la defensa exige la utilización de un amplio y especializado capital humano. Partiendo del amplio cuerpo profesional de la UNED, se ha ido ampliando con los años la participación de profesores pertenecientes a una decena de universidades españolas. A ello hay que añadir la presencia de especialistas del Ministerio de Defensa, que aúnan la doble condición de mandos militares y titulados universitarios; su número alcanza casi una quinta parte del conjunto del profesorado del Instituto.

Los objetivos generales que se pretendieron conseguir con la creación del Instituto Universitario de agruparon en torno a cuatro aspectos principales relacionados con la paz, la seguridad, la defensa y los asuntos militares: desarrollar la investigación científica desde una perspectiva interdisciplinar, fomentar las enseñanzas de posgrado, promover la difusión de obras científicas y crear un marco de reflexión y diálogo.

\section{LA EDUCACIÓN A DISTANCIA EN EL IUGM}

La educación a distancia, que naciera como método para superar las dificultades espaciotemporales de alumnos con dificultad es para acceder a la enseñanza presencial, se ha convertido con el paso del tiempo en un instrumento docente autónomo y con identidad y metodología propias, además de conformar la vanguardia pedagógica experimental. La evolución de las sociedades industriales y posindustriales, necesitadas de un constante reforzamiento y reciclaje de sus conocimientos; y la revolución de los nuevos medios de comunicación, que han permitido la instantaneidad del acceso a la información y borrado las fronteras del espacio, han sido sus más aliados incentivos. La consecuencia inmediata es el crecimiento constante de la nueva educación a distancia, la multiplicación de los modelos docentes y la adaptación de los programas a las nuevas posibilidades.

En su labor docente, el Instituto utiliza desde su inicio la metodología de la enseñanza a distancia de la UNED, que a lo largo de tres décadas se ha ido desarrollando, hasta el punto de convertirla en una de las principales universidades a distancia del mundo. La oferta docente del IUGM se desarrolla completamente a distancia, aun cuando resulta obligada la asistencia a unas sesiones presenciales de presentación, refuerzo y evaluación. A ello se le suma una doble cualidad del alumnado que es necesario tener en cuenta; por una parte, se trata de alumnos plenamente adultos, la mayor parte de los cuales son profesionales, y que realizan los cursos de postgrado y doctorado movidos 
por unos intereses e inquietudes muy precisos; por otra, los alumnos son titulados superiores universitarios y una parte significativa de ellos miembros de las Fuerzas Armadas con titulación igualmente superior; ambas cualidades reportan unos alumnos que poseen ya un buen nivel de rendimiento intelectual, un probado dominio en la práctica del estudio y un buen manejo bibliográfico.

En la educación a distancia resulta una obligación la utilización de todos los medios disponibles para superar la distancia profesor-alumno y potenciar la relación docente-discente. Por esta razón la enseñanza a distancia se ha visto tan favorecida por la revolución de las nuevas tecnologías y su instrumentalización la ha convertido en la vanguardia de la renovación pedagógica; hasta tal punto que las enseñanzas presenciales han tomado parte de esta metodología, depurada para reforzar sus sistemas docentes. El aprendizaje a distancia descansa en dos pilares bási$\cos$, los materiales, en que se concretan los conocimientos que se pretenden transmitir, y los canales de comunicación, que sirven de enlace entre el alumno y el profesor y hacen posible el seguimiento del aprendizaje. Hasta hace relativamente poco tiempo había una gran discusión entre las ventajas e inconvenientes del medio impreso, el medio audiovisual, el medio informático, el vídeo-disco interactivo. Hoy en día todas estas plataformas (medio impreso, el medio audiovisual, al medio informático) han sido superada por el material multimedia e internet, que aúna las ventajas de todos ellos, agiliza la comunicación entre profesor y alumno, abarata los costos de producción y sobre todo permite la incorporación de medios de reforzamiento, direccionalización y retroalimentación.

En este sentido cabe destacar la apuesta decidida del IUGM por intentar proporcionar a sus alumnos todo el material docente en medios digitales. Utilizando la facilidad de acceso, a un ordenador y el dominio extendido en su uso, se han digitalizado, prácticamente, todos los materiales docentes de cada uno de los programas y se le entregan al alumno a comienzo de su curso en soporte de CD. Lejos de cumplir con su aparente imagen de frialdad y distanciamiento, este medio permite una conducción del proceso docente muy superior al material impreso tradicional: mediante la presentación de los temas, la inclusión de ejercicios autocorregibles -o su envío al profesor a través de correo electrónico-, la posibilidad de acceso a las distintas partes del curso dependiendo de factores tan manejables como los resultados de evaluación o las fechas del curso, etc. Por supuesto, este medio engloba todos los anteriores: si el material impreso resultaba la base del modelo anterior, en soporte informático se ve reforzado su valor con la adición de medios audiovisuales e infográficos, con la capacidad de interactuación entre ellos, y con la posibilidad de establecer vínculos entre elementos componentes del material integrado. Al mismo tiempo, este medio se ha manifestado como el idóneo para el diseño y la administración de cursos que por su especialidad y profundidad necesitan de una constante actualización y que por su propia identidad tienen un número de alumnos reducido (no superior a cien), en los que la edición de material impreso resultaría mucho más costosa. Abundando en estas razones, los materiales se han confeccionando siguiendo el modelo de páginas web; esto presenta claras ventajas: por una parte conforma un entorno ya reconocible por el alumno, sin exigirle un conocimiento de nuevas adaptaciones a plataformas completamente ajenas; por otra, se utilizan los programas instalados en todos los ordenadores (Explorer, Firefox, Opera, etc.), lo que hace innecesario la instalación de nuevos programas-motor en el ordenador del alumno; por último, la propia arquitectura interna del material didáctico se integra plenamente en los mecanismos de conformación y comunicación de la plataforma, haciendo que el proceso de estudio, aprendizaje y evaluación se vean enriquecidos con los procesos de retroalimentación.

El segundo aspecto clave de un sistema de educación a distancia son los canales de comunicación entre la institución y los alumnos. La clásica distribución física (en envío postal), posteriormente reforzada con la utilización de la radio y de la televisión, han dado paso a una nueva era en este campo, la era Internet. La revolución de las tecnologías de comunicación a distancia con un único objetivo: transmitir a distancia voz, datos e imágenes, a un coste mínimo. No resulta posible ni parece necesario aquí hacer ninguna apología de Internet; tan sólo constatar su utilización y exponer la experiencia propia. La utilización de la web para el IUGM se ha basado en tres grandes premisa: sencillez, funcionalidad y valor añadido. Se pretende utilizar Internet no sólo para hacerse visible a la comunidad académica internacional en general y a nuestros alumnos en particular, si no aportar en la página web del Instituto elementos propios, hacer rentable y atractiva su visita periódica. Para ello se ha renunciado a 
la utilización de Internet como un escaparate universal, buscando por el contrario la creación de valor añadido; esto se consigue con la puesta a disposición de la comunidad académica (expertos, profesores, alumnos) de estudios, análisis y papeles sobre un conjunto muy amplio de temas relacionados con la Paz, la Seguridad y la Defensa. Con ser importante esto, la proyección en la red de elementos tan valiosos como corpus legislativos, Guías de expertos o Guías de recursos y de Investigación sobre temas de Seguridad Internacional o Resolución Pacífica de Conflictos aún incrementa más su valor de referencia.

\section{LA FORMACIÓN DE DOCTORES Y ESPECIALISTAS universitarios en Seguridad y Defensa}

Un prestigioso psicólogo vienés de la primera mitad del siglo XX hablaba de la "miseria de la docencia" para designar el tiempo malgastado en explicar cosas sabidas a gentes sin interés ocupando un tiempo que no se tiene; dando por entendido que ese tiempo estaría mejor empleado en la investigación de gabinete o laboratorio, estudios de campo o redacción de trabajos que dieran a conocer los resultados alcanzados. Al día de hoy tal idea no serviría sino como mera confesión de incapacidad; aunque resulte especialmente estimulante el campo de la investigación, la reflexión y la creación, resulta no sólo inevitable si no de por sí muy aconsejable complementarlo y contrastarlo con la labor docente, en sus más variadas concepciones. La experiencia propia, contrastada y compartida con los colegas de diversa procedencia y respondiendo a gran variedad de circunstancia e intereses, puede producir una sinergia que conduzca a la mejora general de las ofertas docentes, lo que redundará directamente en beneficio de los centros universitarios y en la calidad de la enseñanza ofertada a los alumnos.

La oferta docente del instituto se divide en dos grandes campos: la formación de doctores, a través del Programa de Doctorado en Paz y Seguridad Internacional; y la formación de expertos universitarios, a través de un conjunto de cursos de posgrado que de forma modular integran el Máster en Paz, Seguridad y Defensa. El Programa docente del Instituto tiene como finalidad primordial el avance de la docencia multidisciplinar y la contribución al desarrollo del conocimiento sobre cuestiones estratégicas relevantes; en su significado más amplio, esto es, el conjunto de procesos implicados en la identificación, la movilización y la aplicación de recursos en paz y en guerra para reforzar la seguridad nacional e internacional. El objetivo es promover el conocimiento, la deliberación y la comprensión pública sobre seguridad, dentro de un pensamiento plural, en especial sobre los asuntos estratégicos europeos, mediterráneos e iberoamericanos.

\section{Programa de doctorado en Paz y Seguridad INTERNACIONAL}

Los Estudios de Tercer Ciclo impartidos por el Instituto están integrados en la oferta de la UNED, ateniéndose a la legislación general de esta Universidad tanto en la confección del programa como en los requisitos necesarios para el alumno que quiera cursarlo. En la actualidad aún persiste el modelo se las últimas décadas -en vías de transformación a corto plazo-: los doctorandos deben obtener 36 créditos, de los cuales un mínimo de 20 créditos corresponden a los cursos de doctorado y los otros 12 créditos corresponden al Trabajo de Investigación; la defensa de este trabajo tiene lugar en la sede del Instituto en sesión pública ante una comisión de profesores doctores.

El Programa de Doctorado en Paz y Seguridad Internacional consta de 32 cursos agrupados en dos grandes áreas: Estudios de la Paz y resolución pacífica de conflictos, y Estudios de Seguridad Internacional. El alumno puede confeccionar su propio tramo curricular cursando específicamente uno de ellos o conformar su período de docencia de forma alternativa de acuerdo con la siguiente clasificación:

Cursos comunes: Las herramientas de la investigación; Métodos de investigación en ciencias sociales; Guerra y paz en el pensamiento contemporáneo; Historia de las relaciones internacionales desde 1945; Las relaciones civiles-militares; Opinión pública y defensa nacional; La organización de las Naciones Unidas; La política europea de España; Seguridad y defensa en un mundo globalizado.

Subprograma de Estudios de la Paz y resolución pacífica de conflictos: Derecho Internacional Humanitario; La intervención humanitaria y el derecho de injerencia; La Corte Penal Internacional; La protección de los derechos 
fundamentales como elemento esencial de la paz; La búsqueda de alternativas pacíficas a los conflictos; Nuevas concepciones de la paz y la seguridad; Las Organizaciones No Gubernamentales.

Subprograma de Estudios de seguridad internacional: Evolución del pensamiento estratégico; Desarme y control de armamentos; Historia militar contemporánea; Economía de la defensa. el caso español; Prospectiva en los estudios de defensa; Armamento y estrategia de destrucción masiva: nuclear, química, biológica, radiológica y misiles; Nuevas amenazas a la seguridad: terrorismo y crimen organizado; Identidad europea de seguridad y defensa; Instituciones de seguridad en el Área Euroatlántica; Política de seguridad española; Política de seguridad de Estados Unidos; Guerrilla y revolución en América Latina; Cooperación y conflictos en Europa del Este; Cooperación y conflictos en el Mediterráneo; Cooperación y conflictos en Oriente Medio.

El profesorado que imparte el Programa de Doctorado es, por exigencias de las materias, muy variado; lo que ha obligado a ampliar su procedencia. Si en un principio el Programa comenzó siendo impartido casi exclusivamente por profesores integrantes del cuerpo docente de la UNED, con el paso del tiempo se han ido ampliando las asignaturas y se ha realizado una labor de selección buscando la excelencia. En la actualidad el Programa está impartido por profesores doctores pertenecientes a ocho universidades distintas, además de oficiales doctores de las Fuerzas Armadas. Dado que su presencia en el Programa de Doctorado atiende a su alta capacidad y especialización, no existe distinción de ningún tipo entre profesores civiles o militares.

El Programa se adscribe a las áreas de conocimiento de Ciencia Política y de la Administración, Derecho Internacional Público y Relaciones Internacionales, Economía Aplicada, Historia Contemporánea, Historia del Pensamiento y de los Movimientos Sociales, y Sociología. En una de esas áreas debe inscribirse la realización de la Tesis Doctoral que culmina el Programa; su realización, tutorizada por un director doctor a elección del alumno (que incluso puede no pertenecer al cuerpo docente del Instituto, en cuyo caso el alumno debe tener un tutor que sí lo sea), no tiene tiempo establecido para ser concluida, si bien se aconseja que no exceda de los cinco años. La Tesis Doctoral debe ser defendida ante un Tribunal examinador compuesto por cinco doctores, siendo su lectura pública. La culminación del programa supone la concesión del título de Doctor en Paz y Seguridad Internacional otorgado por el Ministerio de Educación español a través de la UNED.

\section{Máster Universitario en Paz, Seguridad y Defensa}

El programa modular de especialización en Paz, Seguridad y Defensa se compone de una serie de cursos que permiten obtener de manera independiente los títulos de Experto Universitario y Especialista Universitario. Establecidos de forma piramidal, la obtención de alguno de ellos y el cumplimiento de una serie de requisitos, permite culminar el programa con la obtención del título de Máster Universitario en Paz, Seguridad y Defensa, título propio de la UNED. La transformación de los estudios de posgrado, con la introducción de los criterios de Bolonia y la articulación del Espacio Europeo de Educación Superior, hará que este modelo sufra necesarias transformaciones, si bien la estructura fundamental será muy similar, dado que ya se están utilizando de forma regular dichos criterios.

Los cursos de posgrado del Instituto están estructurados para conseguir tres objetivos específicos: ofrecer una formación especializada sobre los fundamentos de la Paz, la Seguridad y la Defensa, desde una perspectiva particular e interdisciplinar; promocionar el estudio y la investigación en dichas áreas, y dotar de los instrumentos precisos para la aplicación directa y específica de estos conocimientos al análisis de los complejos y cambiantes temas de la seguridad internacional, a la resolución de conflictos y al estudio de la gestión pública en el ámbito de la defensa. El conjunto de títulos que conforman el Máster Universitario en Paz, Seguridad y Defensa es el siguiente: Títulos de Experto Universitario: Selección y Desarrollo de Recursos Humanos en el ámbito de la Defensa; Apoyo a la Decisión en el ámbito de la Defensa; Gestión Pública en el ámbito de la Defensa; Resolución Pacífica de Conflictos; Misiones Humanitarias y Operaciones de paz. En estos títulos deben cursarse los cuatro módulos de los cinco ofertados, durante un período máximo de dos años académicos. Están avalados en 200 horas, equivalentes a 20 créditos.

Títulos de Especialista Universitario: Gestión Pública y Organización en el ámbito de la Defensa; Resolución Pacífica de Conflictos y Mantenimiento de la Paz; Seguridad Internacio- 
nal (integrando los subprogramas de Seguridad y Defensa en el Este de Europa, en América Latina, en el Mediterráneo, y en Estados Unidos); Política Exterior española; Fundamentos de la Paz, la Seguridad y la Defensa. En estos títulos deben cursarse los seis módulos de los ocho ofertados; durante un período máximo de dos años académicos. Están evaluados en 300 horas, equivalentes a 30 créditos.

Título de Máster: Paz, Seguridad y Defensa. Para su obtención es necesario haber cursado dos diplomas de Especialista Universitario, culminando con la presentación de un trabajo de investigación.

Además de estos cursos modulares que integran el máster, se imparten otros cursos de experto o especialista universitario que atienden a cubrir de forma inmediata las demandas de sectores puntuales sobre los ámbitos trabajados en el Instituto. De estos cursos, destacan por la gran aceptación conseguida los cursos de Experto Universitario en Servicios de Inteligencia, Experto Universitario en Cooperación internacional y ayuda Humanitaria y Experto Universitario en Comunicación de la Defensa; así como el recién estrenado curso de Especialista Universitario en Investigación Criminal.

Estos cursos están dirigidos a profesionales que desarrollen su actividad en el campo de las relaciones internacionales, la cooperación y negociación internacional, inteligencia, prensa y comunicación, o deseen en el futuro dedicarse profesionalmente a estas actividades, sea ésta de carácter público o privado y cuyo ámbito de actuación sea nacional, bilateral o en organismos y entidades supranacionales. El formato flexible del programa permite que una persona pueda cursar únicamente aquellos contenidos que necesite para su desarrollo profesional.

Toda la formación se realiza en la modalidad de educación a distancia, con tres jornadas presenciales por año académico con los profesores de cada uno de los módulos de los respectivos cursos; están establecidas tutorías a distancia que son atendidas por teléfono, correo electrónico y charla electrónica. La evolución de los conocimientos adquiridos se realiza a través de pruebas presenciales y trabajos facultativos complementarios.

El material de trabajo, con la excepción de aquellos que se correspondan con publicaciones existentes, se proporciona al alumno en soporte CD. Además de incluir aquellos textos que los profesores consideran oportunos, se ofrece la facilidad para ampliar sus contenidos con la inclusión de los links que les permita enlazar en cualquier momento con textos publicados o reseñas especializadas sobre los últimos datos de la actualidad sobre los que alumnos deben trabajar. Los canales de comunicación, alumno-profesor son, en función de la interacción, tanto el teléfono como sobre todo el correo y charla electrónicos. Lo que no impide que en el desarrollo del curso y para poder hacer uso del adecuado aparato bibliográfico que se ha de manejar, se usen todas las tecnologías de que dispone la UNED y en particular los medios informáticos que facilitan el acceso a fuentes documentales y centros de documentación de todo el mundo.

\section{UN OBJETIVO FACTIBLE: LA MODULARIDAD INTERCENTROS}

Como se ha explicitado en un principio, el objetivo de este trabajo es exponer el modelo docente impartido por el Instituto Universitario General Gutiérrez Mellado, comunicando su experiencia en la formación de doctores y especialistas en Seguridad y Defensa. Sin embargo la experiencia adquirida por el Instituto permite ser más ambiciosos y presentar ante la comunidad académica objetivos que diseñan un innovador escenario operativo. Como se ha visto en la exposición de los Programas de Doctorado y Máster, las posibilidades de modularidad del modelo docente, que permite una mayor capacidad de flexibilidad en la conformación de programas, no tiene por qué estar restringida al desarrollo de un programa en un solo centro. El objetivo que debería perseguirse es la conformación de una red docente en Seguridad y Defensa que cumpla el triple objetivo de compartir el conocimiento, dar una enseñanza de máxima calidad y conseguir el reconocimiento oficial internacional de los títulos otorgados.

Pueden contemplarse dos alternativas que no tienen por qué ser contrapuestas, sino por el contrario pudieran darse de modo complementario. La primera sería la puesta en común de los programas docentes de los centros interesados en conformar esa red, como paso previo para la participación y colaboración conjunta de dos o más centros en programas propios de cada uno de ellos. Esta posibilidad estaría más cercana al modelo de movilidad del profeso- 
rado y permitiría optimizar recursos humanos, al tiempo que incrementaría el valor de los programas participados por profesores de procedencias variadas.

La segunda alternativa es sustancialmente más ambiciosa, pues supone mucho más que un enriquecimiento de lo ya existente para dar paso a nuevos programas y nuevas ofertas docentes. Desde el Instituto se ha realizado una pormenorizada selección del profesorado para impartir el conjunto de asignaturas y materias que se han considerado fundamentales para nuestros programas, contemplando un escenario fundamentalmente nacional; de igual modo, pero ampliando el ámbito geográfico, puede confeccionarse un programa modular que recoja las demandas curriculares de distintos países, realizándose a continuación una selección de expertos internacionales para que la impartan. Esto permitiría a un conjunto de centros especializados en estudios de Seguridad y Defensa ofertar un programa de estudios al más alto nivel internacional.

En esta línea, el Instituto se haya pilotando la constitución de la Comunidad Iberoamericana de Investigación y
Docencia en Paz, Seguridad y Defensa (CIAID). La iniciativa se centra en la creación de un espacio común para el intercambio de información, estudios y experiencias; una plataforma para el fomento de la investigación y la docencia a través de proyectos conjuntos; una entidad representativa que posibilite el más directo acceso a programas de ayuda y fondos públicos y privados incentivadores de este tipo de actividades. El proyecto se orienta hacia la reunión de actuaciones de instituciones y centros con programas consolidados de investigación, difusión y fomento de los estudios sobre Seguridad y Defensa. Los centros que están Ilamados a integrarse en esta comunidad proceden de dos ámbitos muy distintos, pero que de forma natural y afortunadamente con creciente intensidad colaboran directamente: centros universitarios e institutos de investigación y centros de altos estudios militares.

Un final que nos retorna al inicio de estas páginas. Superando los niveles de mera cooperación, la integración de hecho de los ámbitos universitarios y los centros académicos militares.

Recibido: 25 de enero de 2008

Aceptado: 28 de marzo de 2008 